Urologe 2011 · 50:1541-1542

DOI 10.1007/s00120-011-2774-5

Online publiziert: 12. Dezember 2011

(c) Springer-Verlag 2011

\author{
R. Tunder ${ }^{1} \cdot$ M. Goepel ${ }^{2}$ \\ ${ }^{1}$ Health Care Management Institute (HCMI), \\ EBS Universität für Wirtschaft und Recht, EBS Business School, Oestrich-Winkel \\ ${ }^{2}$ Klinik für Urologie und Kinderurologie, Klinikum Niederberg, Velbert
}

\title{
Medizin und Ökonomie sind kein Widerspruch
}

Ein strenger Blick auf die derzeitige Situation vieler Krankenhäuser und Arztpraxen zeigt, dass ihnen die gegenwärtige Lage sehr zu schaffen macht - ganz zu schweigen davon, ob sie überhaupt für die Herausforderungen der Zukunft genügend gewappnet sind. So hört man allen Orten vom Leid der Krankenhäuser wie auch Arztpraxen, sie könnten die Last eines nicht endenden Kostendruckes, eines dringenden Investitionsbedarfs in moderne Medizintechnik und eines sich verschärfenden Ärzte- bzw. Mitarbeitermangels kaum noch stemmen. Der Ausblick in die Zukunft verheißt kaum Besserung. Eine längere Lebenszeit der Menschen, die alternde Gesellschaft und die damit einhergehende Multimorbidität stellen das Gesundheitswesen vor gewaltige kapazitäre und finanzielle Herausforderungen.

Die gegenwärtige Situation zu lösen und die richtigen Weichen für die Herausforderungen der Zukunft zu stellen, ist das Gebot der Stunde. Die Ökonomie kann hierzu eine Hilfestellung bieten. In ihrer Grundfunktion versucht sie mit verschiedenen Theorien, Modellen und Konzepten zwischen den unbegrenzten Bedürfnissen (wie etwa dem Wunsch nach hochwertiger medizinischer Versorgung für jeden) einerseits und den knappen Ressourcen (finanzielle Mittel) und Kapazitäten (Mitarbeiter, Infrastruktur und Medizintechnik) anderseits zu vermitteln. Innerhalb einer sozialen Ordnung geht von den ökonomischen Grundprinzipien eine Lenkungsfunktion aus, die nicht nur den Sicherstellungsauftrag der Gesund- heitsversorgung, sondern auch den dauerhaften Erhalt einer hohen Versorgungsqualität für die Bevölkerung gewährleistet.

Gerade um dem Anspruch an eine hochwertige Medizin für alle gerecht $\mathrm{zu}$ werden, können sich Krankenhäuser und Arztpraxen einer wirtschaftlichen Führung und Verantwortung nicht entziehen.

Die vorliegende Ausgabe widmet sich dem Thema Medizin und Ökonomie. Hierzu hinterfragt der erste Beitrag generell, was die Gesundheitsökonomie zu leisten vermag, um gegenwärtigen und zukünftigen Herausforderungen in der medizinischen Versorgung zu begegnen (Beitrag R. Tunder). Anschließend werden die Markt- und Wettbewerbspositionen fachärztlicher Praxen einem kritischen Blick unterzogen. Diese Auseinandersetzung soll den Dialog zwischen Medizin und Management stimulieren, um sich über die eigenen Leistungspotenziale einerseits und den allgegenwärtigen Marktanforderungen andererseits bewusst zu werden (Beitrag H. Räwer et al.).

In diesem Sinne setzt sich auch der nächste Beitrag mit der Frage auseinander, wie die Leistung von Kliniken und Praxen mit Hilfe von besonderen Ansätzen des Marketings verbessert werden kann. Ein besonderer Fokus wird dabei auf die Analyse dienstleistungsspezifischer Qualität gelegt, um schließlich Implikationen für die Arzt-Patienten-Beziehung hieraus abzuleiten (Beitrag R. Mattmüller und J. Gehbauer). Mit der Beziehung unterschiedlicher Leistungserbrin- ger in Form von Ärztenetzen beschäftigt sich ein weiterer Beitrag. Das Ziel von Ärztenetzen ist es, die Behandlung von Patienten sowohl an der Schnittstelle von haus- und fachärztlicher Versorgung als auch im Übergang zu vor- und nachgelagerten Sektoren zu koordinieren und die relevanten Leistungserbringer miteinander zu verbinden, um damit schließlich die medizinische Versorgung zu verbessern. Interdisziplinäre Netzwerke bilden somit eine wichtige Grundlage für sektorübergreifende Versorgungsformen (Beitrag V. Amelung und S. Wolf).

Eine ureigene ökonomische Fragestellung ist die Bewertung von Aufwand und Ertrag. Übertragen auf das Gesundheitswesen werden die Kosten dem Nutzen einer medizinischen Intervention gegenübergestellt. Einen international vielfach verwendeten, in Deutschland hingegen nicht berücksichtigten Bewertungsmaßstab zur Ermittlung des Nutzwertes therapeutischer Interventionen stellt der QALY-Ansatz dar (Beitrag B. Poulsen Nautrup). In einem weiteren Beitrag erfolgt eine Auseinandersetzung mit den neuesten Anforderungen im Führungsverhalten. Nicht nur die gegenwärtige Situation, sondern auch der allgemeine gesellschaftliche Wandel bringt mit sich, dass Mitarbeiter nicht mehr hierarchisch, sondern konsensbildend geführt werden wollen. Die sog. empathische Führung stellt ein Managementkonzept dar, um eine positive Resonanz mittels einer motivierenden Führungskultur zu erzielen. Mit Hilfe dieses Ansatzes können Führungskräfte sowohl in Krankenhäusern als auch 
in Arztpraxen Veränderungen effizienter steuern und gleichzeitig die Motivation der Mitarbeiter steigern (Beitrag S. Ruhl und U. Wetterauer).

Die Bewertung onkologischer Therapien aus Sicht einer Kasse ist das Thema des letzten Beitrags. Die onkologische Versorgung der Versicherten stellt eine große Herausforderung dar. Die Zuweisungen aus dem Gesundheitsfonds decken die meist hohen Kosten für onkologische Therapien nur teilweise. Deshalb wir die Evaluation der Wirksamkeit und der Kosteneffizienz onkologischer Therapieverfahren immer wichtiger (Beitrag $\mathrm{H}$. Rebscher et al.).

Die Auswahl der Beiträge zeigt, dass ökonomische Fragestellungen in der medizinischen Versorgung kein Widerspruch sind, auch wenn das Spektrum einer möglichen ökonomischen Bezugnahme bei weitem noch nicht ausgeschöpft ist. Wir laden die Leserschaft herzlich ein, den Dialog zwischen Medizin und Ökonomie aufzunehmen und die Verknüpfung nicht als Bedrohung für das Eine, sondern als Chance für das Gesamte zu sehen.

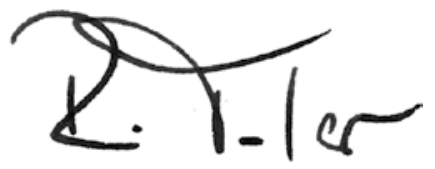

R. Tunder

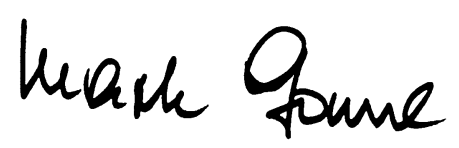

M. Goepel

\section{Korrespondenzadresse}

\section{Prof. Dr. R. Tunder}

Health Care Management Institute (HCMI), EBS Universität für Wirtschaft und Recht, EBS Business School,

Hauptstraße 31, 65375 Oestrich-Winkel ralph.tunder@ebs.edu

\section{Galenus-von- \\ Pergamon-Preis 2011}

\section{Springer Medizin zeichnet exzellente Forschung und ehrenamtliches Engagement aus}

Auch in diesem Jahr hat Springer Medizin den von der Ärzte Zeitung Verlags $\mathrm{GmbH}$ gestifteten Galenus-von-Pergamon-Preis vergeben und würdigt damit exzellente Forschung in Deutschland. 12 unabhängige Experten haben im Oktober 2011 über die Preisträger in den Kategorien Primary Care, Specialist Care und Grundlagenforschung entschieden. Als Schirmherrin des Galenus-von-PergamonPreises lobte die Bundesministerin für Bildung und Forschung, Frau Professor Dr. Annette Schavan, die herausragenden Leistungen aller Forscher-Teams.

Primary Care

Der Preis in der Kategorie Primary Care würdigt ein Medikament, das bei einer breiten Patientengruppe eingesetzt wird. In diesem Jahr hat Amgen/GlaxoSmithKline (GSK) den Preis für Prolia ${ }^{\circledR}$ erhalten. Mit Prolia $^{\circledast}$ steht erstmals ein monoklonaler Antikörper zur gezielten Osteoporosetherapie zur Verfügung. Der Antiköper hemmt die Knochenresorption und schützt somit Frauen in der Postmenopause und Männer mit Prostatakrebs vor Frakturen.

\section{Specialist Care}

Der Preis in der Kategorie Specialist Care zeichnet ein Medikament aus, das zur Behandlung seltener Erkrankungen verwendet wird. In diesem Jahr ist Amgen der Gewinner für Nplate ${ }^{\circledR}$, das erste zugelassene Medikament zur Stimulierung der Thrombozytenbildung. Es ist indiziert zur Behandlung von Erwachsenen mit einer chronischen Immun-(idiopathischen)thrombozytopenischen Purpura (ITP), die auf andere Therapien nicht ansprechen.
Die Preisträger in diesen beiden Kategorien erhielten jeweils eine Medaille und eine Urkunde.

\section{Grundlagenforschung}

In dieser Kategorie wird ein Bewerber prämiert, der eine herausragende wissenschaftliche Arbeit in der pharmakologischen Grundlagenforschung eingereicht hat. Die Auszeichnung ging an Professor Wolfgang Kühn aus Freiburg. Er und sein Team haben sich in der Forschung um die Entschlüsselung molekularer Mechanismen der autosomal dominanten polyzystischen Nierenerkrankung (ADPKD) verdient gemacht. Der Preisträger erhält zur Medaille und Urkunde zusätzlich ein Preisgeld in Höhe von 10.000 Euro.

CharityAward 2011

Mit dem im Jahr 2009 erstmals gestifteten CharityAward zeichnet Springer Medizin jährlich Menschen und Organisationen aus, die sich ehrenamtlich um behinderte, kranke und hilfsbedürftige Menschen verdient gemacht haben. Wer Gewinner wird, entscheiden die Leser der Medien von Springer Medizin. Preisträgerin 2011 ist die in Peru geborene Ärztin Jenny De la Torre: Sie wird für ihren inzwischen 16-jährigen Einsatz für Obdachlose in Berlin-Mitte ausgezeichnet. Im Rahmen dieses Engagements hat sie eine Stiftung gegründet und das Berliner "Gesundheitszentrum für Obdachlose" aufgebaut. Der Award besteht aus einem Medienpaket in einem Wert von 100.000 Euro und einem Barscheck über 50.000 Euro. Schirmherr des Preises ist Bundesgesundheitsminister Daniel Bahr. 